\title{
Post-partum hypertriglyceridemia induced acute pancreatitis
}

\author{
Mahima Arya $^{1 *}$, Sunita Lalwani ${ }^{2}$, Gargee Pore ${ }^{3}$, Aniket Kakade ${ }^{1}$
}

\begin{abstract}
${ }^{1}$ Department of Obstetrics and Gynecology, ${ }^{3}$ Department of Medicine, Bharati Vidyapeeth and Medical College, Pune, Maharashtra, India

${ }^{2}$ Department of Obstetrics and Gynecology, Lalwani Mother and Child Care, Pune, Maharashtra, India
\end{abstract}

Received: 11 September 2020

Accepted: 28 October 2020

\author{
*Correspondence: \\ Dr. Mahima Arya, \\ E-mail: aryamahima424@yahoo.com
}

Copyright: (C) the author(s), publisher and licensee Medip Academy. This is an open-access article distributed under the terms of the Creative Commons Attribution Non-Commercial License, which permits unrestricted non-commercial use, distribution, and reproduction in any medium, provided the original work is properly cited.

\begin{abstract}
Gestational hypertriglyceridemia is well established but is considered an unusual cause of acute pancreatitis with a relatively low incidence. We hereby report a notable triad of hypertriglyceridemia, diabetic ketoacidosis and acute pancreatitis in a woman with 2 months of post-partum status delivered at a private hospital with known case of gestational diabetes mellitus. Presenting with acute abdomen with a surprise on table. Salmon pink coloured blood withdrawn in the vacutainer, turning lactescent post centrifugation. Her serum triglycerides level were $1750 \mathrm{mg} / \mathrm{dl}$, random blood sugar of $870 \mathrm{mg} / \mathrm{dl}$ and total cholesterol of $978 \mathrm{mg} / \mathrm{dl}$. Computerized tomography of abdomen was confirmatory of acute pancreatitis. Patient was treated aggressively with intravenous (IV) fluid resuscitation and IV insulin therapy as per diabetic ketoacidosis (DKA) management protocol. She recovered well and was discharged on day 4.
\end{abstract}

Keywords: Diabetic ketoacidosis, Hypertriglyceridemia, Salmon pink blood, Post-partum, Acute pancreatitis

\section{INTRODUCTION}

Hypertriglyceridemia is a rare cause of acute pancreatitis and accounts for $1-4 \%$ of acute pancreatitis cases. ${ }^{1}$ The incidence of gestational hypertriglyceridemia is relatively low ranging between 3 and 7 in 10,000 cases. $^{2}$ Hypertriglyceridemia presenting as salmon pink blood is uncommon. Serum triglyceride level of $>1000 \mathrm{mg} / \mathrm{dl}$ is needed to trigger an acute attack of pancreatitis. ${ }^{1,3}$ Hypertriglyceridemia can either be primary or secondary to alcohol, pregnancy, insulin resistance/deficiency, poorly controlled diabetes or drugs like tamoxifen, oestrogen and thiazides. ${ }^{1}$

The present case involves postpartum trigger of diabetic ketoacidosis (DKA) and high triglyceride levels which eventually culminated into acute pancreatitis. DKA, pancreatitis and hypertriglyceridemia were successfully resolved by insulin therapy and intravenous (IV) fluids. Need for regular treatment and blood sugar level (BSL) monitoring in a case of postpartum diabetes mellitus is well illuminated.

\section{CASE REPORT}

26 year old second para, delivered 2 months ago by elective caesarean section in view of previous lower segment caesarean section (LSCS), in a private hospital with known case of gestational diabetes mellitus, noncompliant to her oral hypoglycaemic drugs. She came with acute abdomen and 2 episodes of vomiting since 1 day. On arrival she was conscious and obeying commands with stable haemodynamics, maintaining a mean arterial pressure of $65 \mathrm{~mm}$ of $\mathrm{Hg}$ without any vasopressor support. Her saturation on room air was $98 \%$ with a respiratory rate of 16 cycles/min and her systemic and general examination were unremarkable. While blood was being withdrawn for carrying out the baseline investigations, salmon pink coloured blood was surprisingly noticed Figure 3. Her baseline random blood sugar was $870 \mathrm{mg} / \mathrm{dl}$, urine ketones were large $(++++)$ and arterial blood gas $(\mathrm{ABG})$ was 
suggestive of high anion gap metabolic acidosis with hyperlactatemia. Her lipid profile sent was deranged and serum amylase and lipase were elevated. Ultrasonography (USG) whole abdomen showed bulky pancreas with coarse echotexture following which a computerised tomography scan of abdomen (Figure 1,2) was done which confirmed the findings of acute on chronic pancreatitis. The case was then discussed with the intensivist after which she was immediately shifted to the intensive care unit (ICU) for further management. In the ICU she was aggressively treated with fluid resuscitation and IV insulin therapy after confirming baseline potassium values. Serial ABG's, electrolytes and blood sugars were monitored and strict input output charting was done.

She responded well to the above line of management and was discharged on day 4 on oral hypoglycaemic and lipid lowering drugs in a stable condition with laboratory values near normal.

Table 1: Baseline investigations on admission.

\begin{tabular}{|c|c|}
\hline Parameters & Values \\
\hline Random blood sugar (mg/dl) & 870 \\
\hline Serum triglycerides (mg/dl) & 1750 \\
\hline Serum total cholesterol (mg/dl) & 978 \\
\hline Haemoglobin (g/dl) & 16.4 \\
\hline PCV $(\%)$ & 27 \\
\hline Serum lipase (mg/dl) & 4290 \\
\hline Serum amylase (mg/dl) & 363 \\
\hline HBA1c (\%) & 6.8 \\
\hline \multicolumn{2}{|l|}{ ABG } \\
\hline pH & 7.19 \\
\hline $\mathrm{pCO}_{2}$ & 27 \\
\hline Bicarbonate & 8.3 \\
\hline Serum lactates & 1.8 \\
\hline Anion gap & 18 \\
\hline Potassium & 4 \\
\hline Base deficit & -3.0 \\
\hline Serum sodium (meq/l) & 114 \\
\hline Serum chloride (meq/l) & 103 \\
\hline Serum potassium (meq/l) & 4.2 \\
\hline
\end{tabular}

Table 2: Investigations on day 4, prior to discharge.

\begin{tabular}{|ll|}
\hline Parameters & Values \\
\hline Random blood sugar $(\mathbf{m g} / \mathbf{d l})$ & 97 \\
\hline Serum triglycerides $(\mathbf{m g} / \mathbf{d l})$ & 149 \\
\hline Serum total cholesterol (mg/dl) & 188 \\
\hline Haemoglobin $(\mathbf{g} / \mathbf{d l})$ & 12.2 \\
\hline ABG & \\
\hline pH & 7.32 \\
\hline Bicarbonate & 18.3 \\
\hline Serum sodium (meq/l) & 140 \\
\hline Serum chloride (meq/l) & 132 \\
\hline Serum potassium (meq/l) & 4.6 \\
\hline Urine ketones & Negative \\
\hline
\end{tabular}

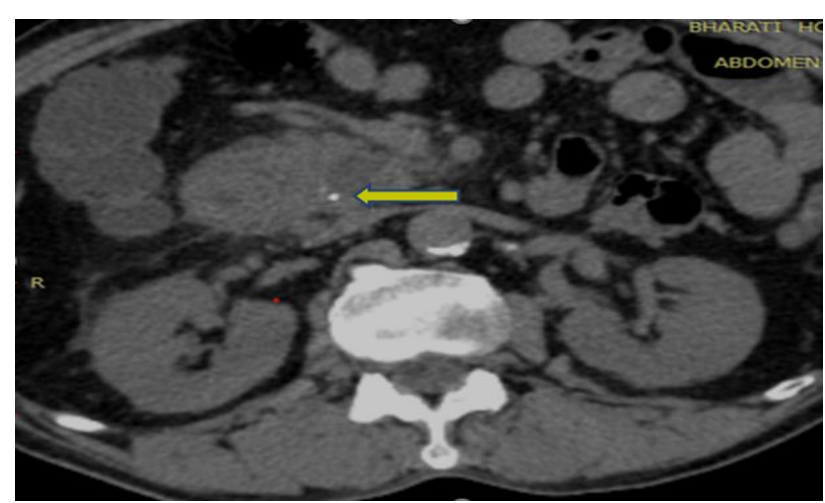

Figure 1: CT scan abdomen showing hyperdense foci in the head of pancreas, suggestive of calcifications.

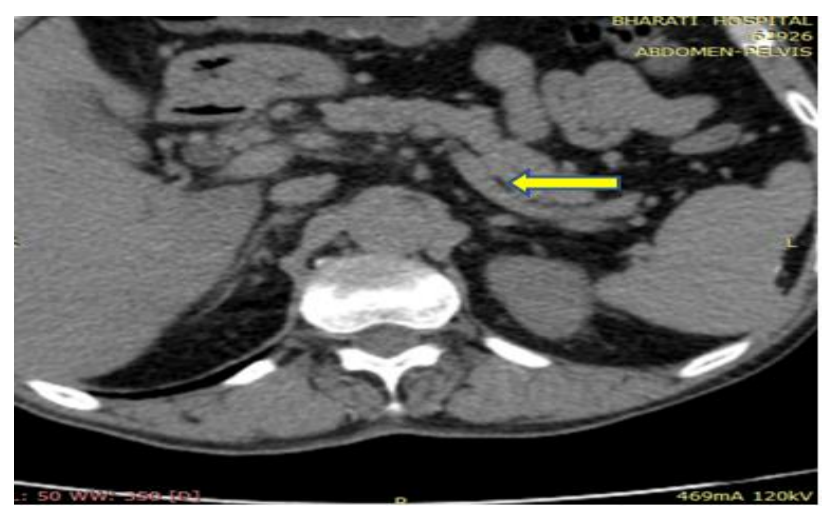

Figure 2: CT scan abdomen showing dilatation of the main pancreatic duct in the body and tail region.

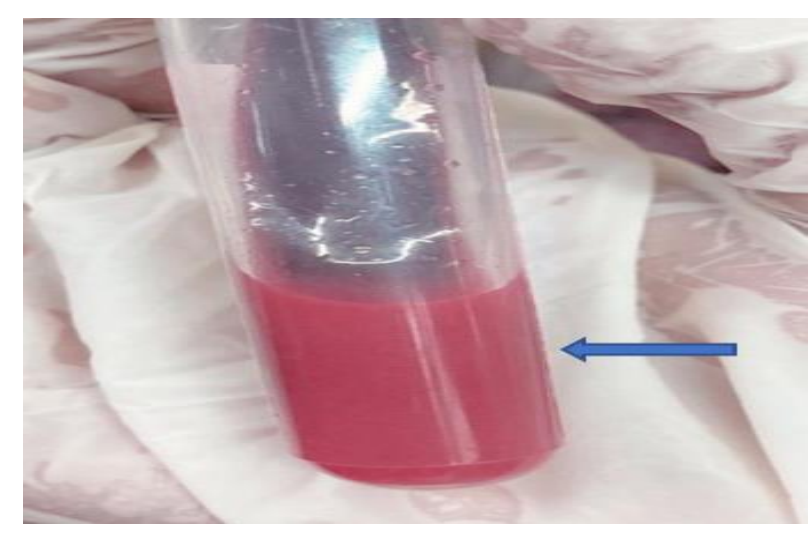

Figure 3: Salmon pink coloured blood which turned lactescent post centrifugation.

\section{DISCUSSION}

We report a case of hypertriglyceridemia induced acute pancreatitis in a post-partum woman with no previous abnormality in lipid profile with non-compliance to antidiabetic treatment. In this case, post-partum pregnant status was considered as an epiphenomenon rather than a cause for development of hypertriglyceridemia. ${ }^{6}$ It is known that pregnancy and puerperium causes physiological estrogen-induced increase in triglyceride rich lipoprotein production and decrease in triglyceride 
clearance due to suppression of lipoprotein lipase action in liver and adipose tissue. ${ }^{6}$ As postulated, hydrolysis of excessive triglyceride can exert radical damage on acinar cells. Also, increased concentration of chylomicrons increases viscosity of blood and can cause capillary obstruction in pancreas. ${ }^{6}$ DKA in pregnancy is a serious but rare complication of hypertriglyceridemia that poses several challenges with respect to diagnosis, management and prevention. Landing in postpartum ketoacidosis concerns an array of complications. A multidisciplinary approach with a critical care emergency is required for maternal stabilization. It should be the first exclusion in mind for any pregnant woman or with post-partum status presenting with abdominal pain, nausea, hypotension, tachycardia, acidotic breathing. The pink discolouration of patient's blood is attributed to the intermingling of opaque triglycerides containing very low-density lipoprotein (VLDL) and chylomicrons with dark red blood corpuscles. ${ }^{4}$ The milky white appearance of centrifuge was mainly due the high level of VLDL or chylomicrons. ${ }^{4}$ Extreme hypertriglyceridemia can result in alteration of peripheral blood and falsely elevated haemoglobin. Acute pancreatitis is a close differential of diabetic ketoacidosis, it can initiate DKA, and DKA itself can mask a co-existing acute pancreatitis that occurs in $15 \%$ of the cases. ${ }^{5}$ So, one should always have a high index of suspicion to diagnose the condition.

\section{CONCLUSION}

Acute pancreatitis should be suspected in all pregnant and postpartum patients admitted for non-obstetric abdominal pain. Symptoms and laboratory investigations can be distorted by physiological changes of pregnancy in view of changes in sex hormones. Therefore, timely diagnosis of acute pancreatitis remains a challenge in such scenario. 6
Computed tomography is a very important modality in diagnosis. Lifestyle modifications such as weight reduction, fat restricted diet and a good control of diabetes with proper compliance is thoroughly mandated.

Funding: No funding sources

Conflict of interest: None declared

Ethical approval: Not required

\section{REFERENCES}

1. Pujar AK, Anil Kumar VR, Sridhar M, Kulkarni SV. An interesting case of hypertriglyceridaemic pancreatitis. J Clinic Diagnost Res. 2013;7(6):1169.

2. Wong B, Ooi TC, Keely E. Severe gestational hypertriglyceridemia: A practical approach for clinicians. Obstet Med. 2015;8(4):158-67.

3. Khan AS, Latif SU, Eloubeidi MA. Controversies in the etiologies of acute pancreatitis. J Pancreas. 2010;11(6):545-52.

4. Tsai DE, Mato A, Porter DL, Vogl DT. Hypertriglyceridemia presenting as "pink blood" and elevated hemoglobin level. Am J Hematol. 2008;83(3):253-5.

5. Vijayavarman V. A rare triad of diabetic ketoacidosis, hypertriglyceridemia and acute pancreatitis: a case report. Int J Res Med Sci. 2019;7(7):2859.

6. Serpytis M, Karosas V, Tamosauskas R, Dementaviciene J, Strupas K, Sileikis A, et al. Hypertriglyceridemia-induced acute pancreatitis in pregnancy. J Pancreas. 2012;13(6):677-80.

Cite this article as: Arya M, Lalwani S, Pore G, Kakade A. Post-partum hypertriglyceridemia induced acute pancreatitis. Int J Reprod Contracept Obstet Gynecol 2020;9:5134-6. 